\title{
Demonstration of Long-Term Thermal Stability of a Silicon-Organic Hybrid (SOH) Modulator at $85^{\circ} \mathrm{C}$ C. Kieninger ${ }^{(1,2) *}$, Y. Kutuvantavida ${ }^{(1,2)}$, J. N. Kemal ${ }^{(1)}$, H. Zwickel ${ }^{(1)}$, H. Miura ${ }^{(4)}$, S. Randel ${ }^{(1)}$, W. Freude ${ }^{(1)}$, S. Yokoyama ${ }^{(3,4)}$, and C. $\operatorname{Koos}^{(1,2) * *}$ \\ (1) Karlsruhe Institute of Technology (KIT), Institute of Photonics and Quantum Electronics (IPQ), Karlsruhe, Germany (2) Karlsruhe Institute of Technology (KIT), Institute of Microstructure Technology (IMT), Karlsruhe, Germany \\ (3) Institute for Materials Chemistry and Engineering, Kyushu University, 6-1 Kasuga-koen Kasuga-city, Fukuoka 816-8580, Japan \\ (4) Department of Molecular and Material Sciences, Kyushu University, 6-1 Kasuga-koen Kasuga-city, Fukuoka 816-8580, Japan Authore-mail address: *clemens.kieninger@kit.edu,**christian.koos@kit.edu
}

Abstract: We demonstrate highly stable silicon-organic hybrid ( $\mathrm{SOH})$ modulators fulfilling

Telcordia standards for high-temperature storage. We show error-free $40 \mathrm{Gbit} / \mathrm{s}$ signaling with drive voltages of $1.65 \mathrm{~V}_{\mathrm{pp}}$ using a device stored at $85^{\circ} \mathrm{C}$ for 2700 hours.

OCIS codes: (250.7360) Waveguide modulators; (230.2090) Electro-optical devices; (130.3120) Integrated optics devices

\section{Introduction}

Efficient electro-optic (EO) modulators are at the heart of optical transceivers used in a variety of networks from short-reach data-center communications to metro and long-haul links. A particularly attractive modulator concept relies on the silicon-organic hybrid (SOH) approach, which combines silicon photonic (SiP) waveguide structures with organic EO materials and thus merges the advanced SiP fabrication technology with the unrivalled EO coefficients of organic materials [1]. SOH modulators have shown outstanding performance in terms of modulation efficiency [1,2] and achievable data rates [3] proven, e.g., by recent demonstrations of 16QAM transmission at a symbol rate of $100 \mathrm{GBd}$ [4]. However, one of the remaining challenges is the long-term stability of SOH devices at elevated temperatures, limited by thermally induced relaxation of the poled organic EO materials. In this paper, we report on the first demonstration of long-term stable $\mathrm{SOH}$ modulators, fulfilling Telcordia standards for hightemperature storage [5], requiring that the modulators retain $85 \%$ of the EO activity when stored at $85{ }^{\circ} \mathrm{C}$ for more than $2000 \mathrm{~h}$. This is achieved by employing a recently introduced side-chain EO polymer [6] with adamantyl side groups, which increase the glass transition temperature $\left(T_{\mathrm{g}}\right)$ to $172^{\circ} \mathrm{C}$. The viability of the device is demonstrated by generating error-free $40 \mathrm{Gbit} / \mathrm{s}$ on-off-keying (OOK) signals using a device which was stored at $85^{\circ} \mathrm{C}$ for $2700 \mathrm{~h}$.

\section{Device fabrication and characterization}

The SOH Mach-Zehnder modulator (MZM) concept is shown in Fig. 1(b). Each MZM arm comprises an SOH phase shifter consisting of a Si slot waveguide. The slot waveguide is formed by two parallel $240 \mathrm{~nm}$-wide and $220 \mathrm{~nm}$ high Si rails which are separated by a $190 \mathrm{~nm}$ wide slot filled with the EO material, see Fig. 1(a) for the chemical structure. Thin $n$-doped Si slabs connect the rails to a ground-signal-ground (GSG) transmission line. This geometry results in a large overlap of the radio frequency (RF) mode and the optical mode in the slot region, leading to highly efficient EO modulation. The waveguide structure is fabricated on standard silicon-on-insulator (SOI) wafers in a SiP foundry using $248 \mathrm{~nm}$ deep-UV lithography, while the EO material is deposited by an in-house post-processing step. An average acentric molecular orientation and thus an appreciable macroscopic EO activity is achieved by poling: We heat up the chip close to the glass transition temperature $T_{\mathrm{g}}$ of the EO material and apply a poling voltage $U_{\text {pol }}$ across the floating ground electrodes of the MZM, which induces poling fields (green arrows) for aligning the dipolar molecules. Then the device is cooled down while $U_{\text {pol }}$ is maintained. The MZM allows push-pull operation as a drive voltage $U_{\mathrm{d}}(t)$ will induce electric fields (red arrows) that are parallel to the poling direction in one arm and antiparallel in the other arm. The experimental setup for measuring the $\pi$-voltage $U_{\pi}$ is shown in Fig. 1(c). We optically feed the MZM with an external cavity laser (ECL) while we apply a triangular electrical waveform with a peak-to-peak amplitude $U_{\mathrm{d}, \mathrm{pp}}>U_{\pi}$ provided by a function generator (FG). The light is detected by a photo diode (PD) connected to an oscilloscope which also measures the drive voltage. $U_{\pi}$ can thus be directly extracted as the drive voltage difference needed to switch the MZM from maximum to minimum transmission, see Fig. 1(d).

The poling-induced average acentric orientation of the EO molecules represents an energetically unfavorable state leading to a thermally induced reorientation and resulting in an increase of $U_{\pi}$. For a systematic investigation of this process we pole four nominally identical $1.5 \mathrm{~mm}$-long SOH MZMs and store them in an oven at a temperature of $85^{\circ} \mathrm{C}$ in accordance with pertinent Telcordia standards [5]. The modulators were removed from the oven from time to time to monitor the increase in $U_{\pi}$. The results are summarized in Fig. 1(e), where $U_{\pi}$ normalized to its respective initial value is plotted as a function of time. All devices show qualitatively the same trend: After an initial burn-in of approximately $300 \mathrm{~h}$, the devices retain over $85 \%$ of their modulation efficiency, fulfilling Telcordia standards for high-temperature storage. As indicated by the dashed lines, the data show fairly good agreement with an adapted Debye model [7] of the form $U_{\pi}(t) / U_{\pi}(0)=1 /(a+b \exp (-t / \tau))$, where $t$ is the time and $a=0.7$, $b=0.3$ and $\tau=250 \mathrm{~h}$ are typical fitting parameters. The increase in $U_{\pi}$ may be attributed to the release of stress in 

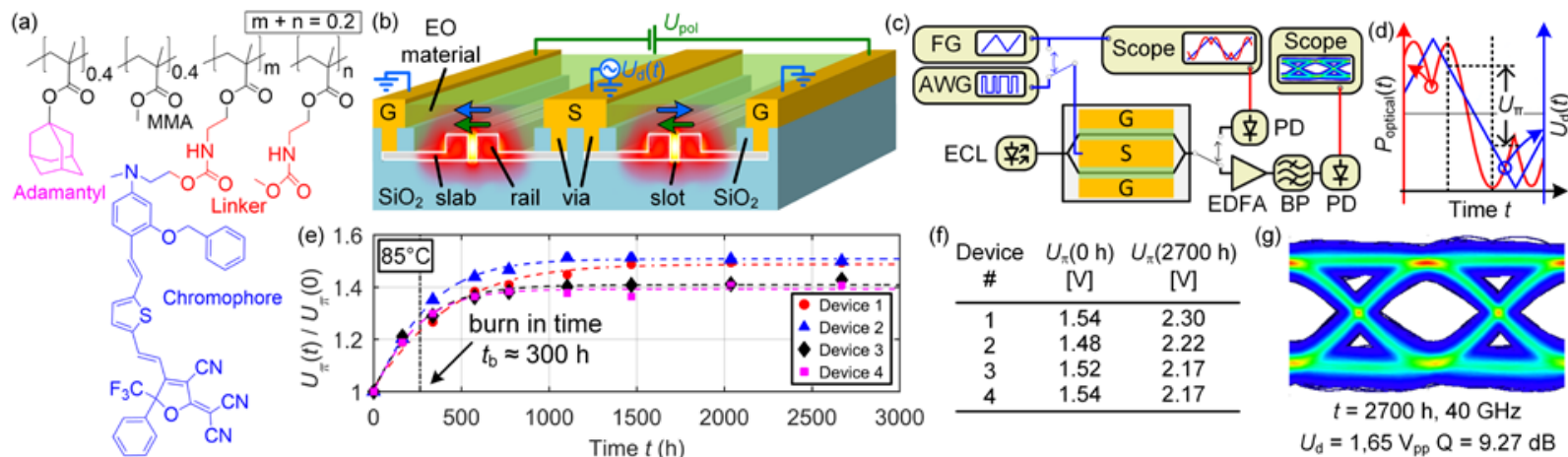

Fig.1. SOH-modulator concept and characterization. (a) Chemical structure of the employed side-chain EO polymer containing an adamantyl group, which increases $T_{\mathrm{g}}$ to $172{ }^{\circ} \mathrm{C}$. (b) SOH MZM cross section. Each arm comprises a Si slot waveguide clad by the EO material. For poling, we apply a voltage $U_{\text {pol }}$ across the floating ground electrodes at elevated temperature. This aligns the dipoles of the EO material (green arrows). We remove $U_{\text {pol }}$ after cooling down which freezes this alignment. A drive signal $U_{\mathrm{d}}(t)$ leads to electric fields (blue arrows) that are parallel (antiparallel) to the poling orientation in the left (right) arm, hence enabling push-pull operation. (c) Setup for $U_{\pi}$ characterization and data transmission. A low-speed function generator (FG) or a high-speed arbitrary waveform generator (AWG) is used to drive the MZM. We couple light from an external cavity laser (ECL) to the MZM via grating couplers. The slowly modulated light is detected by a photo diode (PD) for measuring $U_{\pi}$. For high-speed operation, the modulated light is amplified and detected by a PD for generating the eye diagram. (d) $U_{\pi}$ measurement. (e) Normalized $U_{\pi}$ as a function of time for four $1.5 \mathrm{~mm}$ long $\mathrm{SOH}$ devices stored at $85^{\circ} \mathrm{C}$. After an initial increase during the first hundreds of hours, $U_{\pi}$ reaches a stable value. (f) Absolute $U_{\pi}$ measurements at $t=0,2700 \mathrm{~h}$. (g) Eye diagram for OOK signaling at $40 \mathrm{Gbit} / \mathrm{s}$ with a device stored at $85^{\circ} \mathrm{C}$ for $2700 \mathrm{~h}$. After post-equalization, the Q factor amounts to $9.27 \mathrm{~dB}$, indicating error-free transmission. the EO polymer induced during the cooling step of the poling process. This effect can generate free volume (voids) in the EO material, which facilitates the reorientation of the molecules. The stable value of $U_{\pi}$ after its initial increase can be attributed to the large difference between $T_{\mathrm{g}}$ and the storing temperature of $85^{\circ} \mathrm{C}$, at which the molecular mobility of the polymer chain is negligible. To the best of our knowledge, this is the first demonstration of longterm thermal stability of SOH MZM. The absolute values for $U_{\pi}$ at $0 \mathrm{~h}$ and $2700 \mathrm{~h}$ are summarized in Fig. 1(f), indicating consistent performance. Note that the devices with a length of $L=1.5 \mathrm{~mm}$ exhibit an average modulation efficiency $U_{\pi} L$ of only $3.3 \mathrm{Vmm}$ after $2700 \mathrm{~h}$ at $85^{\circ} \mathrm{C}$. This is roughly 4 times more efficient than other stable organic EO modulators [8,9]. Given the vast potential of theory-guided material optimization, we expect that even more efficient long-term stable SOH devices with $U_{\pi} L$ products well below $1 \mathrm{Vmm}$ will come into reach.

\section{Data transmission}

The viability of these MZM for high-speed modulation is demonstrated in a data transmission experiment, see Fig. 1(c) for the experimental setup. We apply a pseudo-random bit sequence of length $2^{15}-1$, obtained from an arbitrary waveform generator (AWG) to an MZM aged for $2700 \mathrm{~h}$ at $85^{\circ} \mathrm{C}$. The optical carrier provided by an external cavity laser is coupled to and from the device using grating couplers. The fiber-to-fiber loss amounts to $16 \mathrm{~dB}$ and is compensated by amplifying the modulated light with an EDFA. A band pass filter (BP) is used to suppress noise before detecting the light in a photo diode connected to a real-time oscilloscope. We apply a post equalizer on the detected data and obtain the eye diagram in Fig. 1(g) for a data rate of $40 \mathrm{Gbit} / \mathrm{s}$ and a peak-to-peak drive voltage of $1.65 \mathrm{~V}_{\mathrm{pp}}$. The measured Q-factor amounts to $9.27 \mathrm{~dB}$, which suggests error-free transmission.

\section{Summary}

We have shown that SOH MZM employing an EO material with a large $T_{\mathrm{g}}$ fulfill Telcordia standards for high temperature storage. The devices outperform competing organic-based modulators [8,9] in terms of modulation efficiency and show great potential for further improvement. In addition, we proved the high-speed performance of $\mathrm{SOH}$ modulators even after high temperature storage by generating error-free $40 \mathrm{Gbit} / \mathrm{s}$ OOK signals. We believe that these results pave the way towards industrial applications of SOH devices.

Funding: ERC Starting Grant 'EnTeraPIC' (\# 280145), ERC Grant 'TeraSHAPE' (\#773248), Alfried Krupp von Bohlen und Halbach Foundation, Helmholtz International Research School for Teratronics (HIRST), Karlsruhe Nano-Micro Facility (KNMF)

\section{References}

1. C. Kieninger et al., "Ultra-High In-Device Electro-Optic Coefficient of $\mathrm{r}_{33}=390 \mathrm{pm} / \mathrm{V}$ Demonstrated in a Silicon-Organic Hybrid (SOH) Modulator," arXiv:1709.06338 (2017).

2. S. Wolf et al., "DAC-Less Amplifier-Less Generation and Transmission of QAM Signals Using Sub-Volt Silicon-Organic Hybrid Modulators," J. Light. Technol. 33, 1425-1432 (2015).

3. S. Wolf et al., "Silicon-Organic Hybrid (SOH) Mach-Zehnder Modulators for 100 Gbit/s On-Off Keying," arXiv:1709.01793 (2017).

4. S. Wolf et al., "Coherent modulation up to 100 GBd 16QAM using silicon-organic hybrid (SOH) devices," ArXiv:1710.00056 (2017).

5. Telcordia, "GR-468-CORE: Generic Reliability Assurance Requirements for Optoelectronic Devices Used in Telecommunications Equipment," (2004).

6. F. Qiu et al., "An electro-optic polymer-cladded $\mathrm{TiO}_{2}$ waveguide modulator," Appl. Phys. Lett. 1091, 173301-123302 (2016).

7. P. Debye, Polar Molecules, (The Chemical Catalog Company Inc., 1929).

8. H. Miura et al., "High thermal stability $40 \mathrm{GHz}$ electro-optic polymer modulators," Opt. Express 25, 28643 (2017).

9. R. Dinu et al., "Environmental Stress Testing of Electro-Optic Polymer Modulators," J. Light. Technol., JLT 27, 1527-1532 (2009). 